University of Wollongong

Research Online

Faculty of Engineering and Information

Faculty of Engineering and Information

Sciences - Papers: Part A

Sciences

$1-1-2014$

Power allocation algorithm in OFDM-based cognitive radio systems

Van Vinh Nguyen

Hung Yen University of Technology and Education, vinhnv.utehy@gmail.com

Yang Shouyi

Zhengzhou University

Le Chung Tran

University of Wollongong, Ictran@uow.edu.au

Follow this and additional works at: https://ro.uow.edu.au/eispapers

Part of the Engineering Commons, and the Science and Technology Studies Commons

Research Online is the open access institutional repository for the University of Wollongong. For further information contact the UOW Library: research-pubs@uow.edu.au 


\title{
Power allocation algorithm in OFDM-based cognitive radio systems
}

\author{
Abstract \\ In orthogonal frequency division multiplexing (OFDM)-based cognitive radio (CR) systems, the optimized \\ algorithms for sub-carrier power allocation face the problems of complex iterative calculation and difficult \\ realization. In this paper, we propose an exponential power distribution function and derive a sub-optimal \\ power allocation algorithm. This algorithm aims to allocate power of in-band subcarriers of cognitive \\ users according to the numerical characteristics of the power distribution function by using a convex \\ optimization numerical method under linear constraints. This algorithm has the advantages of fast \\ calculation speed and easy realization, and reduces the interference to the authorized users, which is \\ caused by the power leakage of the in-band subcarriers of cognitive users to the out-of-band subcarriers. \\ Simulation results show that the proposed algorithm maximizes the inband channel capacity of the \\ cognitive users under certain interference thresholds of the authorized users, thus increasing their \\ transmission rate.
}

Keywords

power, ofdm, cognitive, algorithm, allocation, radio, systems

Disciplines

Engineering | Science and Technology Studies

\section{Publication Details}

N. Van. Vinh, Y. Shouyi \& L. Chung. Tran, "Power allocation algorithm in OFDM-based cognitive radio systems," in 2nd IEEE International Conference on Computing, Management and Telecommunications (ComManTel 2014), 2014, pp. 13-18.

This conference paper is available at Research Online: https://ro.uow.edu.au/eispapers/2295 


\section{Power Allocation Algorithm in OFDM-Based Cognitive Radio Systems}

\author{
Nguyen Van Vinh ${ }^{1,2}$ \\ ${ }^{1}$ Hungyen University of Technology \& Education \\ Hungyen, Vietnam \\ ${ }^{2}$ School of Information Engineering \\ Zhengzhou University \\ Zhengzhou, China \\ Email: vinhnv.utehy@gmail.com
}

\author{
Yang Shouyi ${ }^{2}$ \\ ${ }^{2}$ School of Information Engineering \\ Zhengzhou University \\ Zhengzhou, China \\ Email: iesyyang@zzu.edu.cn
}

\author{
Le Chung Tran ${ }^{3}$ \\ ${ }^{3}$ University of Wollongong \\ Australia \\ Email: lctran@uow.edu.au
}

\begin{abstract}
In orthogonal frequency division multiplexing (OFDM)-based cognitive radio (CR) systems, the optimized algorithms for sub-carrier power allocation face the problems of complex iterative calculation and difficult realization. In this paper, we propose an exponential power distribution function and derive a sub-optimal power allocation algorithm. This algorithm aims to allocate power of in-band subcarriers of cognitive users according to the numerical characteristics of the power distribution function by using a convex optimization numerical method under linear constraints. This algorithm has the advantages of fast calculation speed and easy realization, and reduces the interference to the authorized users, which is caused by the power leakage of the in-band subcarriers of cognitive users to the out-of-band subcarriers. Simulation results show that the proposed algorithm maximizes the inband channel capacity of the cognitive users under certain interference thresholds of the authorized users, thus increasing their transmission rate.
\end{abstract}

Keywords-Orthogonal Frequency-Division Multiplexing (OFDM); Cognitive Radio (CR); Power Allocation; Linear Water-Filling; Convex Optimization.

\section{INTRODUCTION}

Due to the rapid growth of wireless communication, the available resources of wireless spectrums are becoming scarcer, impeding the application of new techniques. Cognitive radio (CR) has been widely accepted as an effective method to improve wireless spectrum utilization [1]. It can perceive and detect the dynamic changes of idle wireless spectrums, and make the unused spectrum available to cognitive users. The $\mathrm{CR}$ system must not affect the normal communications of the license users (LUs). The cognitive users (CUs) can communicate with each other by automatically searching and utilizing the idle spectrum. Therefore, CR is the most effective way of solving the problem of spectrum scarcity [2].

As a universally acknowledged technology of the next generation of broadband wireless communication networks, orthogonal frequency division multiplexing (OFDM) technology can improve significantly the performance of cellular systems by utilizing the characteristic of multiuser diversity to distribute the sub-channels, bits and power effectively, and is regarded as an ideal alternative technology of realizing CR systems [3]. CUs can neatly fill the idle spectrum left by LUs, by taking advantage of the OFDM technology. The fast Fourier transform (FFT) module in the receiver can be used for spectrum perception.

Regarding to OFDM-based CR systems, the authors in [4] studied the mutual interference caused by the nonorthogonality between CUs and LUs using the convex optimization theory. The authors in [5] examined the resource allocation plan in an OFDM-based CR network. According to the traditional power allocation scheme (such as water-filling algorithm), more power should be distributed to the sub carrier with a higher quality channel. The authors in [6] proposed an unequal bit-loading algorithm for a non-contiguous OFDM-Based CR system. In fact, such an interference limited scenario limits the transmit power as well as the achievable transmission rate of $\mathrm{CR}$ users. Hence, the design problem is that, given an interference threshold prescribed by the primary users, how much power each CR user's subcarrier should have, so that the transmission rate of CR users could be maximized. In [7], the authors proposed an optimal power allocation scheme using Lagrange formulation. This scheme maximizes the downlink transmission rate of CUs, while keeping the interference induced to the primary users below a threshold. However, the total power constraint was not considered in this paper.

In this paper, we propose a modified power allocation function mentioned in [7]. In particular, we propose an exponential power distribution function, rather than a linear function, with the purpose of optimizing the channel capacity at the CU band. Simulation shows that our proposed sub-optimal power allocation algorithm provides the channel capacity being better than that in [7], and is close to the optimal channel capacity.

This paper is organized as follows. Section II presents the system model and formulates the studied problem as a convex optimization problem. In Section III, we propose a sub-optimal power allocation algorithm, which is based on an exponential distribution function, and examine its two particular cases. Section IV provides the numerical simulations and discussion, and Section $\mathrm{V}$ concludes the paper. 


\section{SYSTEM MODEL}

This text considers the downlink of a CR system. According to how the IEEE 802.11a system model [4] utilizes the OFDM modulation technique, we suppose the disposition of LU band and the idle spectrum which $\mathrm{CU}$ insert is shown in Fig.1.

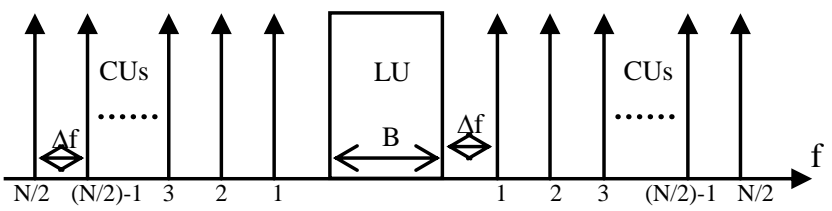

Fig. 1. Distribution of license and cognitive users

Suppose that the LU's bandwidth is $B$, and CU is separated on either side of LU. The interval of every subcarrier in the CU band, and the interval between LU band and $\mathrm{CU}$ band are both $\Delta f$. The sum of subcarriers in the band of $\mathrm{CU}$ is $N$. Because the OFDM modulation mode is used in both the CUs and LU (the attenuation characteristic of power spectral density side lobe), the LU and CUs both interfere with each other.

\section{A. Interference Introduced by Cognitive User's Signal}

According to S. Haykin [1], the power density spectrum (PSD) of the $i_{t h}$ subcarrier in CR user band can be written as

$$
\varphi_{i}(f)=P_{i} T_{s}\left(\frac{\sin \pi f T_{s}}{\pi f T_{s}}\right)^{2}
$$

where $P_{i}$ denotes the transmitting power of subcarrier $i$ in the $\mathrm{CU}$ band, $T_{s}$ denotes the symbol period of OFDM. Therefore, the interference power of the $i^{\text {th }}$ subcarrier in the $\mathrm{CU}$ band to the LU band is:

$$
I_{i}\left(d_{i}, P_{i}\right)=P_{i} \times T_{s} \int_{d_{i-B / 2}}^{d_{i+B / 2}}\left(\frac{\sin \pi f T_{s}}{\pi f T_{s}}\right)^{2} d f
$$

where $d_{i}$ denotes the nearest spectrum distance between the subcarrier $i^{\text {th }}$ in the CU band to the LU band. We denote

$$
K_{i}^{l}=T_{s} \int_{d_{i-B / 2}}^{d_{i+B / 2}}\left(\frac{\sin \pi f T_{s}}{\pi f T_{s}}\right)^{2} d f
$$

Eq. (2) can be written as

$$
I_{i}\left(d_{i}, P_{i}\right)=P_{i} \times K_{i}
$$

\section{B. Interference Introduced by License User's Signal}

The interference power of LU signal after the M-fast Fourier transform (FFT) processing to subcarrier $i$ in $\mathrm{CU}$ band is:

$$
I_{L U}\left(d_{i}, P_{L U}\right)=T_{s} \int_{d_{i-B / 2}}^{d_{i+B / 2}} \varphi_{L U}(f) d f
$$

where $\varphi_{\mathrm{LU}}(\mathrm{f})$ denotes the PSD of the LU signals, and $P_{L U}$ denotes power of the LU signals.

\section{Channel Throughput Analysis of CUs [7]}

Assuming that each subcarrier goes under frequency flat fading and the instantaneous fading gains are perfectly known at the transmitter. The transmit power adaptively loaded in each CR user's subcarrier. With an ideal coding scheme, the transmission rate at the $i^{\text {th }}$ subcarrier, $R_{i}$ for the transmit power, $P_{i}$ and channel fading gain $h_{i}$ is connected via the Shannon capacity formula and is given by [7]:

$$
R_{i}=\log _{2}\left(1+\frac{\left|h_{i}\right|^{2} \times P_{i}}{\Gamma \times \sigma_{i}^{2}}\right)
$$

where $\sigma_{\mathrm{i}}^{2}$ is the sum power of Gaussian noise and the interference caused by PUs on the $i^{\text {th }}$ subcarrier. $\Gamma$ is a constant signal-to-noise ratio (SNR) gap, according to [8], $\Gamma$ has a relationship with the required bit error rate (BER):

$$
\Gamma=\frac{\ln (5 B E R)}{1.5}
$$

\section{OPTIMAL POWER ALLOCATION}

In this section, we propose an optimal power allocation algorithm and investigate its two suboptimal power allocation algorithms, which are less complex and easier to be realized. Note that the optimal CU's channel capacity can be found based on the following optimization problem

$$
\begin{aligned}
& C=\max _{P_{i}} \sum_{i=1}^{N} R_{i}\left(P_{i}, h_{i}\right) \\
& \text { s.t. }\left\{\begin{array}{c}
\sum_{i=1}^{N} I_{i}^{1}\left(d_{i}, P_{i}\right) \leq I_{t h}^{1} \\
\sum_{i=1}^{N} I_{i}^{2}\left(d_{i}, P_{i}\right) \leq I_{t h}^{2} \\
P_{i} \geq 0
\end{array}\right.
\end{aligned}
$$

where $C$ is the CU's channel capacity, $N$ is the total number of subcarriers in the CU band, $R_{i}$ is the transmission rate at the $i^{\text {th }}$ subcarrier, and the interference thresholds prescribed by the left and right primary bands are equal, e.g. $I_{t h}^{1}=I_{t h}^{2}=I_{t h}$.

\section{A. Optimal Power Allocation Scheme}

For the above optimization problem, although the objective function is a concave function, the constraint condition is linear. Thus, the optimization problem is a convex optimization problem, and the convex optimization approach can be used to analyze the optimal power allocation scheme. We use Lagrange multiplier method to construct the Lagrange function:

$$
\begin{gathered}
L\left(P_{i}, \lambda_{1}, \lambda_{2}\right)=\sum_{i=1}^{N} R_{i}\left(P_{i}, h_{i}\right)-\lambda_{1} . \\
\left(\sum_{i=1}^{N} I_{i}^{1}\left(d_{i}, P_{i}\right)-I_{t h}^{1}\right)-\lambda_{2}\left(\sum_{i=1}^{N} I_{i}^{2}\left(d_{i}, P_{i}\right)-I_{t h}^{2}\right)
\end{gathered}
$$

where $\lambda_{1}$ and $\lambda_{2}$ are the Lagrange constraints, $\lambda_{1}, \lambda_{2} \geq 0$.

Therefore, the derivative of Eq.(10) is 


$$
\frac{\partial L}{\partial P_{i}}=\frac{1}{\frac{\delta^{2}}{\left|h_{i}\right|^{2}}+P_{i}}-\lambda_{1} \frac{\partial I_{i}^{1}}{\partial P_{i}}-\lambda_{2} \frac{\partial I_{i}^{2}}{\partial P_{i}}
$$

where

$$
\delta_{i}^{2}=\delta^{2}+I_{L U} ; K_{i}^{l}=\frac{\partial I_{i}^{l}}{\partial P_{i}}=T_{S} \int_{d_{i}-B / 2}^{d_{i}+B / 2}\left(\frac{\sin \pi f T_{S}}{\pi f T_{S}}\right)^{2} d f
$$

The right side of Eq.(11) is equal to zero. Thus, the optimal transmit power can be obtained as:

$$
P_{i}^{*}=\left(\frac{1}{\lambda_{1} k_{i}^{1}+\lambda_{2} k_{i}^{2}}-\frac{\delta_{i}^{2}}{\left|h_{i}\right|^{2}}\right)^{+}
$$

where $(\mathrm{x})^{+}=\max (0, \mathrm{x})$ and Lagrange multiplier $\lambda_{1}, \lambda_{2}$ can be Eq.(13) are obtained:

$$
\left\{\begin{array}{l}
\sum_{i=1}^{N} I_{i}^{1}\left(d_{i}, P_{i}^{*}\right)=I_{t h} \\
\sum_{i=1}^{N} I_{i}^{2}\left(d_{i}, P_{i}^{*}\right)=I_{t h}
\end{array}\right.
$$

From equation (13), we can see that the power is assigned to the $i_{t h}$ carrier of the CU band may appear less than zero. Therefore, here carried out the processing and analysis steps iterative partitioned water filling (IPW) algorithms. After much choice and change of operator until the allocated power on each carrier is non-negative and positive in the CU band [9].

\section{B. Suboptimal Schemes}

By using the above scheme, we can calculate the optimal power allocation policy that maximizes the transmission capacity of the CR user while keeping the interference introduced to the Pos below the specified threshold. However, the complexity of the optimal scheme is high and hence, in the following section, we propose suboptimal schemes based on heuristics that have lower complexity.

In the scheme optimized power allocation, the number of carriers in the $\mathrm{CU}$ band needs to be adjusted from left to right are $N / 2,(N / 2)-1, \ldots, 3,2,1,1,2,3, \ldots,(N / 2)-1, N / 2$, where $N$ is an even number; Similarly when $N$ is odd. In [7] proposed optimization scheme A and B. The authors proposed schemes $\mathrm{C}$ and $\mathrm{D}$, based on power allocation functions exponential.

\section{1) Scheme $C$}

In this scheme, determination of the maximum power can be allocated in each subcarrier is different. We divide the total interference on the number of the available subcarriers, and equal interference threshold per subcarrier will be determined. In this way the maximum power that can be allocated to each subcarrier will be:

$$
P_{i}^{C}=p \times i^{1.5}, i=1,2,3, \ldots, N
$$

From (13) and (14), the maximum power can be written as

$$
P_{i}^{C^{*}}=\frac{i^{1.5} \times I_{\text {th }}}{\sum_{i=1}^{N} i^{1.5} \times K_{i}}
$$

\section{2) Scheme D}

The classic OFDM loading algorithms: uniform power loading and water filling schemes are suboptimal for such an interference-limited scenario as they do not have any constraint on the interference. Therefore, for a given interference threshold $I_{t h}$, power allocated to the $i^{\text {th }}$ subcarrier with the proposed exponential power loading can easily expressed as:

$$
P_{i}^{D}=p \times i^{3.0}, i=1,2,3, \ldots, N
$$

From (13) and (16), the maximum power can be written as

$$
P_{i}^{D *}=\frac{i^{3.0} \times I_{t h}}{\sum_{i=1}^{N} K_{i} \times i^{3.0}}
$$

\section{Simulation Results}

In our simulations, we suppose the values of $T_{s}, \Delta f$ and $B$ to be $4 \mu \mathrm{s}, 0.3125 \mathrm{MHz}, 0.3125 \mathrm{MHz}$ respectively. The total number $N$ of sub-carriers in CU channel is 12 , which means there are six subcarriers in each side of the LU channel (it is similar when $N$ is an odd number). Power of the channel corresponding to each subcarrier in the LU band is created randomly within $[1,10] \mu \mathrm{W}$. The white Gaussian noise in the LU channel is $10 \mu \mathrm{W}$, the interference threshold $\left(I_{t h}\right)$ is $3.2 \mathrm{~mW}$. The total transmitting power of the $\mathrm{CU}$ channel is $1 \mathrm{~W}$. The BER $=10^{-3}$ is considered in all simulations except the last one. Perfect channel state information (CSI) is

\begin{tabular}{|c|c|c|c|c|c|c|c|c|c|c|c|c|}
\hline \multirow{2}{*}{ Scheme } & \multicolumn{12}{|c|}{ Subcarrier power $(\mu \mathrm{W})$} \\
\hline & 1 & 2 & 3 & 4 & 5 & 6 & 7 & 8 & 9 & 10 & 11 & 12 \\
\hline Opt. scheme & 356.92 & 211.25 & 109.14 & 71.08 & 37.36 & $3.2 \mathrm{e}^{-17}$ & $3.3 \mathrm{e}^{-17}$ & 28.35 & 60.09 & 111.25 & 211.25 & 361.42 \\
\hline Scheme A & 36.66 & 30.55 & 24.44 & 18.33 & 12.22 & 6.11 & 6.11 & 12.22 & 18.33 & 24.44 & 30.55 & 36.66 \\
\hline Scheme B & 117.16 & 67.98 & 35.47 & 22.46 & 12.78 & 2.20 & 2.20 & 12.78 & 22.46 & 35.47 & 67.98 & 117.16 \\
\hline Scheme C & 67.97 & 50.77 & 35.53 & 22.42 & 11.72 & 3.86 & 3.86 & 11.72 & 22.42 & 35.53 & 50.77 & 67.97 \\
\hline Scheme D & 137.42 & 85.54 & 47.78 & 22.66 & 7.89 & 1.30 & 1.30 & 7.89 & 22.66 & 47.78 & 85.54 & 137.42 \\
\hline
\end{tabular}
assumed at both transmitter and receiver.

Fig. 2 presents the power distribution histograms of the aforementioned five power distribution methods [10]. Intuitively, a good power allocation will allocate more power to both sides of the $\mathrm{CU}$ band to overcome the interference effect from the LU band.

TAB. I. SUBCARRIER POWERS IN DIFFERENT POWER DISTRIBUTION 


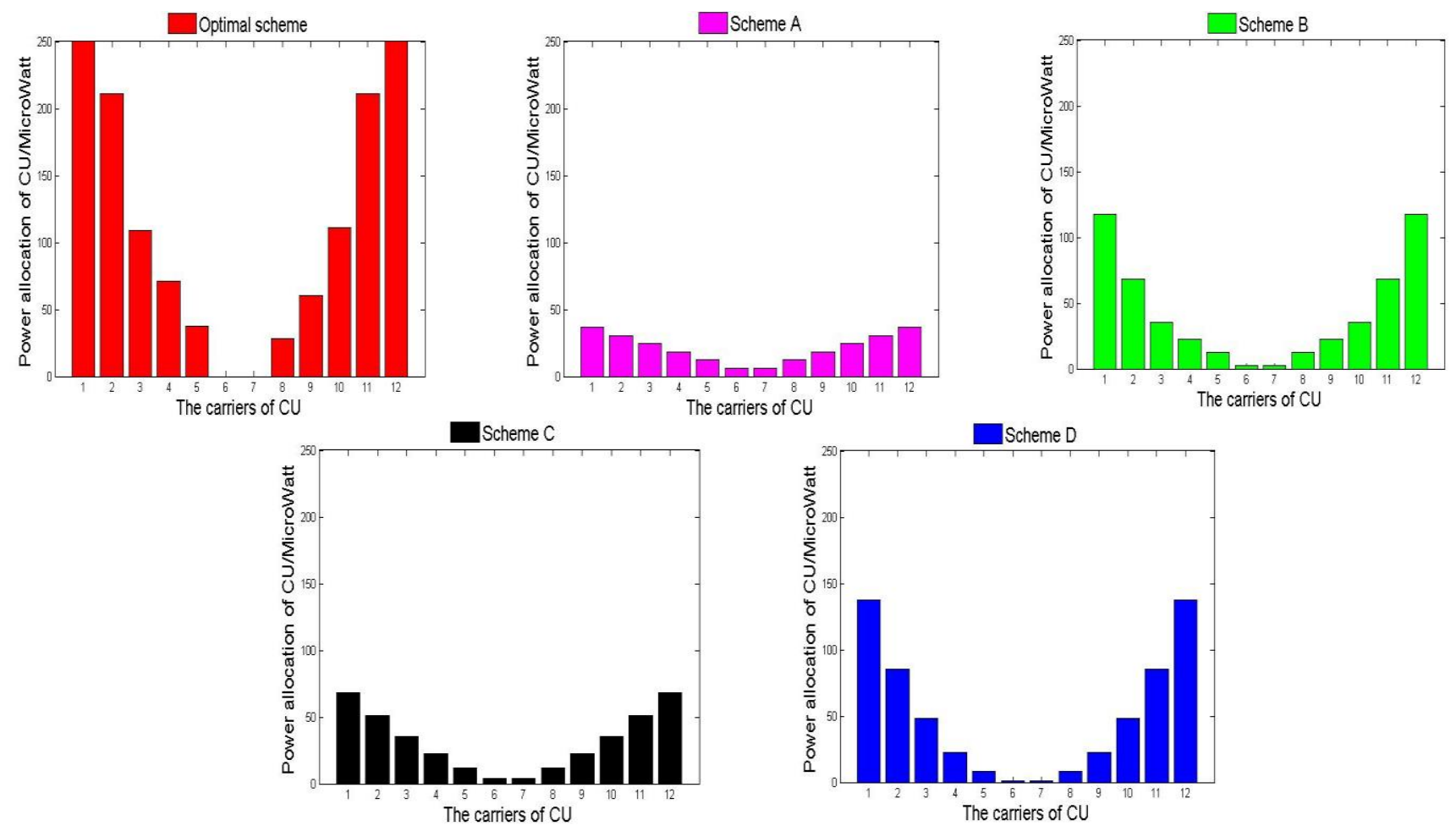

Fig.2. Power distribution histograms of different schemes

Table I summarizes quantitatively the power distribution per subcarrier of the five schemes shown in Fig. 2. Clearly, the transmission powers of the sub-carriers in the $\mathrm{CU}$ band are inversely proportional to the distance to the LU band.

Fig. 3 presents one example realization of the channel power (i.e. $\left|h_{i}\right|^{2}$ ) corresponding to the 12 subcarrier of the CU band. These values are $0.6005,1.3403,0.5153,3.0103$, $0.5603,0.3555,0.0384,0.2870,0.3011,0.8396,0.4553$, and $0.2194 \mathrm{~dB} \mu$ respectively. Again, these values are random created within the range $[1,10] \mu \mathrm{W}$.

In Fig. 4, we plot the achievable capacity of CUs versus the interference threshold introduced to the primary user band for different schemes. We can see that capacity in all schemes increases as the interference threshold increases. In addition, the channel capacity of Scheme D is very close to the channel capacity of the optimal power allocation scheme, while having lower complexity. The channel capacity in Scheme D is higher than that in the two schemes A and B proposed in [7].

Fig. 5 presents the transmit power of the CUs versus the interference threshold introduced to the primary user's band for various schemes. Clearly, the optimal scheme allows us to transmit higher power than the other schemes for a given interference threshold, as the optimal scheme considers interference in its power loading policy for a given interference threshold. Again, it can be observed that, for a given interference threshold, the transmission power in the proposed scheme D is close to that in the optimal scheme, and is significantly higher than that in the two schemes A and $\mathrm{B}$.

In Figures 6 and 7, we examine the channel capacity of the CU channels in our proposed suboptimal scheme D in comparison with that in the optimal power allocation scheme, using the same simulation parameters and conditions mentioned in the previous simulation results, except that the exponent, denoted as $x$, takes different values 1.0, 1.5, 2.0, 2.5, 3.0, and 3.5. In particular, Fig. 6 presents the channel capacity of CUs in our proposed power allocation scheme D with different exponential distributions with different values of the exponent, while Fig. 7 magnifies Fig. 6 for the two example ranges of interference thresholds $1.45 \leq \mathrm{I}_{\text {th }} \leq 1.55$ and $2.95 \leq \mathrm{I}_{\text {th }} \leq 3.05$. From Figs 6 and 7 , one can see clearly that the proposed scheme D with the exponent of 3 has the best performance for the whole considered range of interference thresholds.

Fig. 8 presents the channel capacity corresponding to different values of BER $=10^{-2}, 10^{-3}, 10^{-4}, 10^{-5}$, and $10^{-6}$. Other parameters are kept the same as above. We can see that the channel capacity increases significantly when the $\mathrm{BER}$ requirements are more relaxed. The channel capacity at $\mathrm{BER}=10^{-2}$ almost doubles the channel capacity at $\mathrm{BER}=$ $10^{-5}$. 


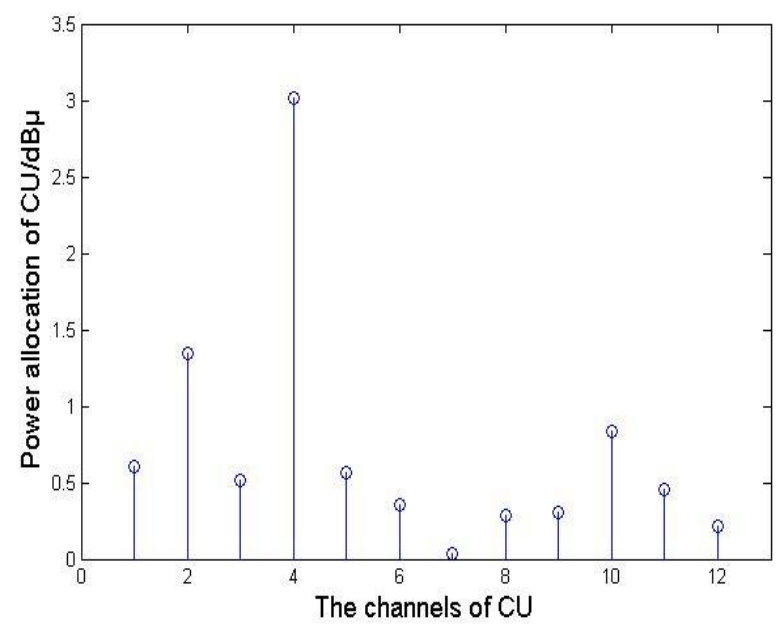

Fig.3. Powers of CU channels

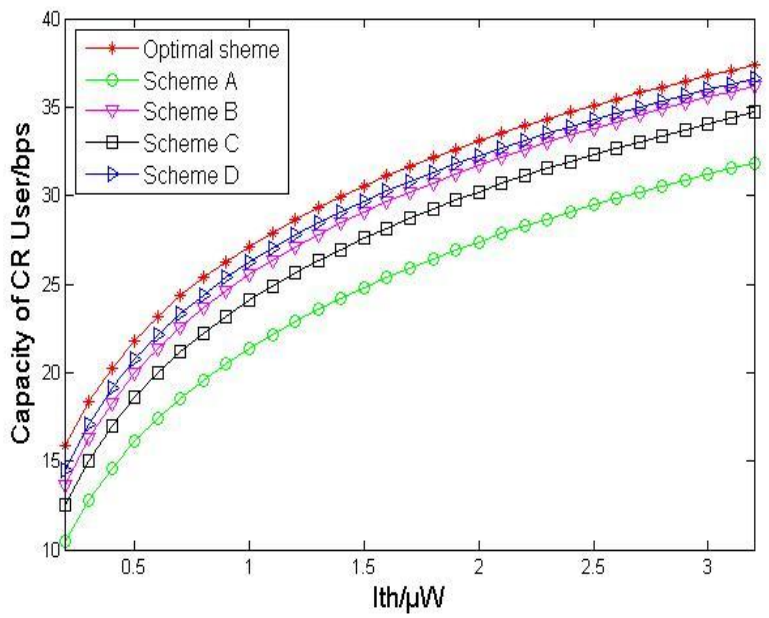

Fig.4. Maximum transmission rate of CUs under different interference thresholds

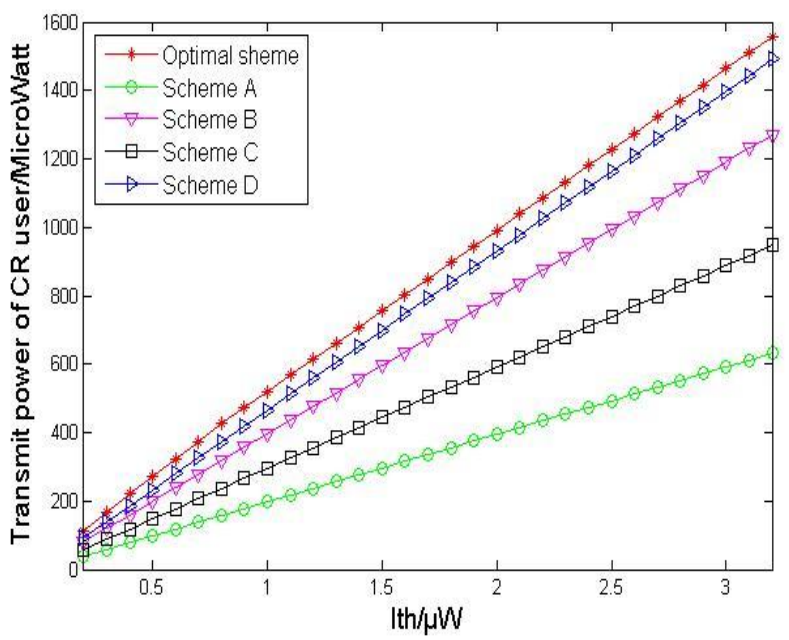

Fig.5. Maximum transmission power of CUs under different interference thresholds

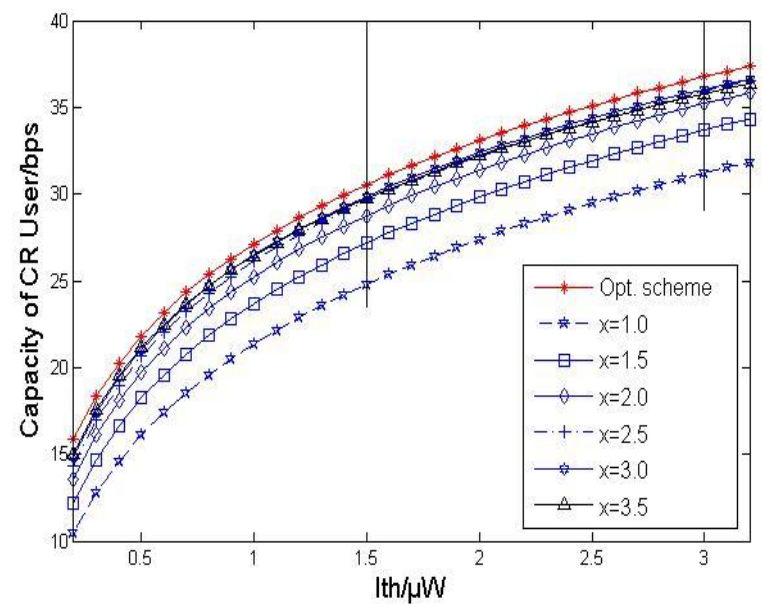

Fig.6. Maximum transmission rate of CUs under different power exponent values
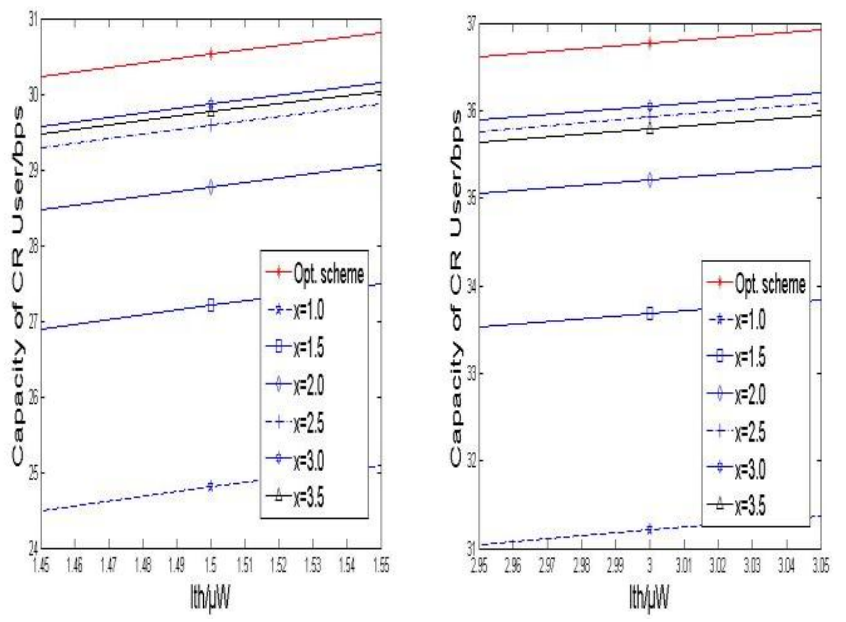

Fig.7. Magnification of Fig.6 in the interference threshold ranges $1.45 \leq \mathrm{I}_{\mathrm{th}} \leq 1.55$ and $2.95 \leq \mathrm{I}_{\mathrm{th}} \leq 3.05$

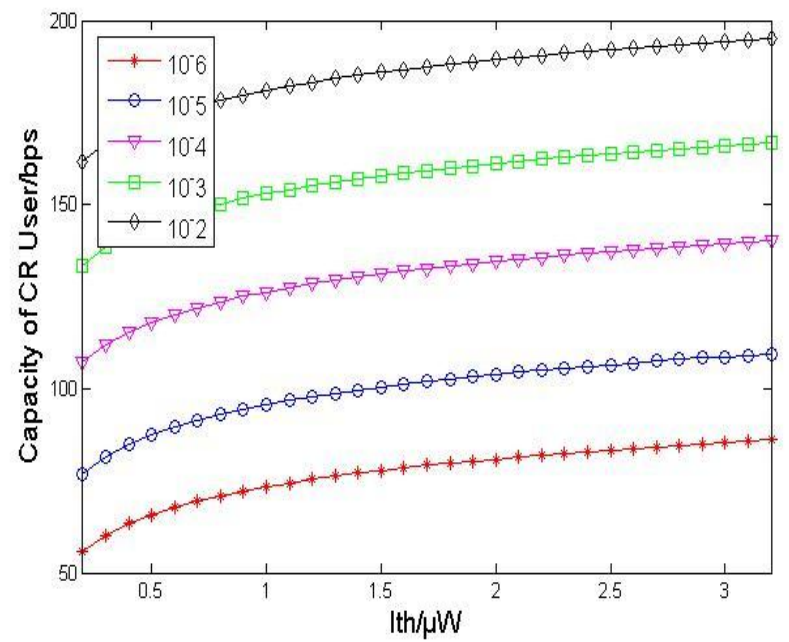

Fig.8. Channel capacity vs. power interference threshold of CUs with different BERs from $10^{-6}$ to $10^{-2}$ 


\section{CONCLUSIONS}

In this paper, we have examined the effect of the interference from the $\mathrm{LU}$ band to the $\mathrm{CU}$ band, and proposed the novel exponential power distribution, rather than the linear power distribution as mentioned in [7]. We have pointed out that the sub-optimal power allocation scheme in an OFDM-based cognitive system, which achieves the CU band channel capacity close to the optimal one, could be found by solving a convex optimization problem. We also examined the $\mathrm{CU}$ band channel capacity with different exponent values and realized that our proposed scheme D corresponding to the exponent value of 2.5 provides a higher channel capacity than the schemes in [7]. Our future work could be the further consideration of sub-optimal power allocation algorithms under the effect of LU and CU guard bands.

\section{REFERENCES}

[1] S. Haykin, Simon, Cognitive radio, "Brain-Empowered Wireless Communications," IEEE Journal on Selected Areas in Communications, vol. 23, pp. 201-220, Feb. 2005.

[2] Akyildiz I. F., Won-YeoI Lee, Vuran Mehmet C., Mohanty S., "Next generation, dynamic spectrum access, cognitive radio wireless networks, a survey," Computer Networks of Elsevier, vol. 46, pp. 4048, April. 2008.
[3] Weiss T. A, Jondral F. K, Spectrum pooling, "An innovative strategy for the enhancement of spectrum efficiency," IEEE Communications Magazine, vol. 42, pp. S8-14, Mar. 2004.

[4] Weiss T, Hillenbrand J, Krohn A, Jondral, F.K, "Mutual interference in OFDM-based spectrum pooling Systems," Proc. IEEE Vehicular Technology Conference Spring, IEEE Press, vol. 4, pp1873-1877, 2004.

[5] G. Bansal, M. J. Hossain, V. K. Bhargava, "Optimal and Suboptimal power allocation schemes for OFDM-based cognitive radio systems," IEEE Transactions on Wireless Communications, vol. 7, pp. 47104718, Nov. 2008.

[6] Wyglinski, Alexander M., "Effects of bit allocation on noncontiguous multicarrier-based cognitive radio transceivers," Proc. IEEE Veh. Technol. Conf., pp. 1-5, Sept. 2006.

[7] G. Bansal, M. J. Hossain, V. K. Bhargava, "A daptive power loading for OFDM-based cognitive radio systems," Proc. IEEE ICC, IEEE Press, pp. 5137-5142, 2007.

[8] G. J. Foschini and J. Salz, "Digital communications over fading radio channels," Bell Syst. Tech. J., pp. 429-456, Feb. 1983.

[9] Wang P, Zhao M, Xiao L, "Power allocation in OFDM-based cognitive radio systems", Proc. IEEE Global Communication Conference, IEEE Press, pp. 4061-4065, 2007.

[10] Nguyen Van Vinh, Yang Shouyi, "Optimal Power Allocation and Power Contrain in OFDM-Based Cognitive Radio Systems," Proc. IEEE Conference on World Congress on Information and Communication Technologies (WICT'13), Hanoi, Vietnam, 15-18 Dec 2013. 